\title{
A Novel Link Break Distance (LBD) and Pre- emptive Threshold Alarm (PTA) based Link Repair Approach for MANET
}

\author{
Poorva Shukla \\ Department of Computer Science \& Engineering \\ IES-IPS Academy, Indore, (M.P), India
}

\author{
Neeraj Shrivastava \\ Department of Computer Science \& Engineering \\ IES-IPS Academy, Indore, (M.P), India
}

\begin{abstract}
Mobile ad hoc network is a temporary wireless network having movable mobile nodes as the only element of network. For transmission of data individual nodes behaves as a router. A during this process of routing operations the motion of nodes \& environmental factors like traffic, delays etc causes the link break occurs between the nodes \& data is lost. AODV is an on demand distance vector routing protocol. This work focuses on improving the performance $\&$ fault tolerant routing in MANET. It has two mechanism named as source repair \& local repair for dealing with this link repair. But the decision is very complicated that which algorithm is applied at what instance. Node does not know the triggering condition of starting those algorithms. Also when this link break occurs during the data exchange then loss of data occurs. So there must be mechanism which is able to inform us prior the condition of link failure.
\end{abstract}

This work proposes a novel Link Break Distance \& Preemptive Threshold Alarm (LBT-PTA) mechanism for this decision making based on prior information. This information will give the continuous assessment of link condition. We define some triggering points at which decision is made based on threshold value. Also the hop count index according to distance is calculated to apply source or local repair algorithm. Initial experimental analysis shows that the proposed approach is going to provide effective results than any other existing approaches.

\section{Keywords}

MANET, AODV, Link Break, LBD (Link Break Distance), PTA (Pre-emptive Threshold Alarm), Source Repair, Local Repair.

\section{INTRODUCTION}

Mobile ad hoc network (MANET) is a challenging area of work because its adaptation property in any type of area, where it is complicated to establishes an infrastructure. It provides a transmission without any infrastructure components. Instead of that mobile nodes will perform the same functionality of those components. These are of type WSN, Zigbee \& Bluetooth. In infrastructure less wireless networks, there is no need to use a base station. Each node acts as a router. Conventional protocols of wired networks are not suited for ad hoc networks. Hence, there is a need to design new dynamic protocols with changing topologies to work in wireless medium [1]. Current research work is heavily oriented towards designing better algorithms, as well as removing the limitations of the previous ones. Such works always remove some sort of problems but help develop other limitations as side effect. According to the platform on which they are developed, routing algorithms can be categorized as: Pro-active \& Reactive.

Routing in MANET is an active optimization problem as the explore space change over time. They must be capable of applying shrinking method to adjust with new network variables [2]. The routing strategy is defined as the rule that specifies what node to take next at each decision node to reach the destination node. It requires secure rte optimization mechanism as proposed in [3] for better route. Due to these better secure routes, the time varying nature of the topology of the networks, traditional routing techniques such as distancevector and link-state algorithms that are used in fixed networks, cannot be directly applied to mobile ad hoc networks. The constraints of MANETs demand the need of specialized routing algorithms that can work in a decentralized and self-organizing way. The routing protocol of a MANET must dynamically adapt to the variations in the network topology \& can be classified into two major categories - Proactive and Reactive [4]. The proactive or table driven routing protocols maintain routes between all node pairs all the time. It uses periodic broadcast advertisements to keep routing table up-to-date. This approach suffers from problems like increased overhead, reduced scalability and lack of flexibility to respond to dynamic changes. The reactive or on-demand approach is event driven and the routing information is exchanged only when the demand arises. The routing is initiated by the source.

During this routing decisions so many environmental problem arises which causes the performance of network to be degraded. These problems are mobility aware secure routing [5], QoS requirements [6] congestion control, flow control, link break, shortest path, malicious activity detection, \& so many others [7]. Among all of them the fault tolerance characteristics is achieved by only avoiding the link failures which is a major functionality of MAC layer. In MANET, ADOV has some of their algorithm already dealing with those failures. These algorithms are source repair \& local repair. But still they are not able to take the decision regarding the time of applying either algorithms. Also this link repair can occurs after certain conditions so it must be analyzed before to pre-empt the failure. This work focuses on that pre-emption by LBD-PTA proposed methodology.

\section{BACKGROUND}

Effective routing can be considered as important activity in MANET. The main function of a routing algorithm is to find an initial path between a source and a destination, and then to maintain data forwarding between the two nodes.

Routing transmission of data from source to destination comes under the MAC layer protocol. They must provide various 
characteristics like fault tolerance \& parameter aware routing. Some of the consideration must also made towards a dynamic energy conscious routing algorithm ACER where cross layer interaction is provided to utilize the energy related information from physical and MAC layers [8]. MAC protocols can be broadly classified as contention-based and contention-free schemes. In general, contention-based MAC protocols have good throughput and delay performance under light load conditions, but their performance degrades as the load increases because of the increased collisions, retransmissions, and control message overhead [9]

Although there have been many reactive routing protocols proposed in the literature, but to accomplish the proposed work will uses reactive protocol AODV. It is an on-demand, single path, loop-free distance vector protocol. It combines the on-demand route discovery mechanism in DSR with the concept of destination sequence numbers from DSDV. However, unlike DSR which uses source routing, AODV takes a hop-by-hop routing approach. AODV offers quick adaptation to dynamic link conditions, lower processing needs and memory overhead, lower network utilization, and determines unicast routes to destinations. It uses destination sequence numbers to ensure loop freedom at all times, avoiding problems associated with classical distance vector protocols. It enables dynamic, self-starting, multi-hop routing between participating mobile nodes wishing to set up and maintain an ad hoc network. AODV allows mobile nodes to obtain new routes speedily for new destinations.

AODV offers quick convergence even in the case of path breakage. When paths breaks, all the nodes which are on the side of the source are conveyed message that path breaks has occurred and delete the related entries from the routing table In AODV, when a node (source) requires a connection to another node (destination), a global route discovery operation is initiated by the source, resulting in a flooding of Route Request (RREQ) messages in the network. When (at least) one of these messages is received by the intended destination or by a node which has a fresh enough route to the destination, a Route Reply (RREP) message is sent back to the originator of the route discovery process. As the RREP packet travels back towards to the originator of the request, each node along the route inserts next hop information into its routing table for the destination requested. Once the source node receives the RREP message, it can start to forward data towards the destination along the route established.

\section{Review of Local Link Repairing Protocol}

As mentioned above, one of the key roles and challenges of ad hoc network routing protocols is to deal with link failures, and to repair routes in these situations. How quickly and efficiently routes can be repaired determines to a large extent the overall performance of MANETs and WMNs. AODV has two basic route repair approaches to deal with link failures. Routes can either be repaired by re-establishing a new route from scratch starting from the source node called as source repair, or they can be locally repaired by the node that detects the link break along the end-to-end path called as local repair. In some situations source repair will lead to better performance, in other situations local repair will be the more appropriate choice.

One of the key challenges in this context, which to the best of our knowledge has not yet been thoroughly investigated, is the question of when to apply which of the two route repair strategies. Standard AODV, as defined in [3], uses a fixed hop count based threshold as a basis for deciding when to invoke Local Repair or Source Repair in case of a link break.

With the help of above mentioned control packets AODV initiate route request on demand by sending RREQ and get available path by RREP. Freshness of route is maintained with the help of sequence number. Error in route is detected by hello message; in case of route error RERR message is generated by the node detecting route failure. Original AODV does not exploit the fast and localized partial route recovery method. It does not have any mechanism to find alternate path (figure 1) in between the transmission link. Many other researchers worked in the direction of route maintenance if it has link breakage during the transmission of data [5].

We are going to develop an on-demand, multipath distance vector routing protocol for mobile ad hoc networks. Specifically, we propose multipath extensions to a wellstudied single path routing protocol known as ad hoc ondemand distance vector (AODV) [6]. The protocol guarantees loop freedom and selection of alternate paths.

In future Performance comparison of our proposed route repair with AODV using ns-2 simulations shows that AODV is able to effectively cope with mobility-induced route failures. In particular, it reduces the packet loss by up to $40 \%$ and achieves a remarkable improvement in the end-to-end delay (often more than a factor of two). Our proposed mechanism will also reduce routing overhead by about $30 \%$ by reducing the frequency of route discovery operations

\section{RELATED STUDY}

In 2011 S R. Azzuhri, M Portmann, W L Tan proposes a novel decision based method for link repairing \& identification of repairing algorithm. According to this AODV uses a fixed Local Repair threshold in terms of number of hops from the destination where a link break occurs, to determine if to invoke Source Repair or Local Repair. This Paper define a Local Repair Threshold parameter that determines how far along the end-to-end path that a link break needs to occur in order to initiate Local Repair, as opposed to Source Repair [1]. The simulation results show that the optimal choice of the Local Repair Threshold, in terms of Packet Delivery Ratio depends on the network load

In 2011 Jyoti Jain et. al. in [4] gives a general survey of research on local repair of link, if it is broken during communication for MANET and proposes a new local repair scheme in order to make up the deficiency of the existing local repair schemes. The improved local repair scheme concerns about the over head requirement and end to end delay in transmission. Nodes are required to keep the next two-hop node address for each route entry in routing table. During local repair, the repairing node use Ant algorithm for finding new route for next to next node in the link considering that other part of the link is already in existence. Reduced size of F-ANT and B-ANT will give significant reduction in overhead [4]. In this case repairing proposed algorithm will be highly adaptive, scalable and efficient and mainly reduces end-to-end delay in high mobility cases.

In 2010 Zhiming Xu et. al. proposes an approach based on the ODMRP (On-Demand Multicast Routing Protocol) in MANET (Mobile Ad hoc Network), a reliable ODMRP (RODMRP) for preferable throughput and especially suited for high-speed MANET, which includes packet acknowledgement, lost packet recovery, secure authentication and QoS based packet delivery [10]. With the exploration of active network, R-ODMRP constructs the multicast routing 
based on the cluster, establishes a distributed mechanism of the acknowledgment and recovery of packet delivery. The performance of the proposed schemes is evaluated based on the network simulator and achieves a significant improvement.

In this paper [11] author S. Subburam et. al. proposes a novel approach of routing to support for quality of service (QoS), such as delay and bandwidth constraints. To provide quality of service, extensions can be added to the AODV routing protocol while finding the route. These extensions specify the service requirements which must be met by nodes rebroadcasting a route request (RREQ) or returning a route reply (RREP) for a destination. Moreover, the proposed routing protocol will follow the concept of unicast-type two hop local route repair protocol to recover the lost links efficiently while increasing network reliability, increasing utilization, minimizing the number of control messages and shortening the repair delay.

In 2005 Hui-Yao et. al. proposed A Cluster-Based Multipath Dynamic Source Routing in MANET (CMDSR) that is designed to be adaptive according to network dynamics [12]. It uses the hierarchy to perform Route Discovery and distributes traffic among diverse multiple paths. The CMDSR is based on a 2-level hierarchical scheme: the 1-cell cluster and 2-server cluster. The main idea of this proposition is to transfer the Route Discovery procedure to the 2-server level to prevent the network flooding due to the DSR Route Discovery. Thus, Route Discovery does not require flooding mechanism and overhead is minimized and improve the networks scalability. The route overhead increases significantly with an increasing node density and a significant number of nodes in the plane structure, so the scalability is hard.

In paper [13] S. Revathi et. al. suggested that the route shortening can be incorporated with route repairing mechanism, to improve the performance of the AODV. The route shortening scheme works by replacing some redundant nodes in the active route, with a node that is not on the active route. If there is a link failure between the two nodes, the route repairing mechanism repairs the route, by using the nodes that are close enough to the route to overhear the message. Whenever the links go down, the DRSR replaces the failed links with the optimum route that is adjacent to the main route and not sending and RRER message to the source node to initiate the route discovery process again.

In this paper the author Jianlin Guo et. al. we present a loopfree routing protocol in LLNs based on IETF RPL framework. The proposed routing protocol defines rank as proper fraction to guarantee no routing loops can be created. A DODAG local repair method is also proposed for fast route repair in [14].

In 2003 Srdjan Krco et. al. proposed Improved Neighbor Detection Algorithm for AODV Routing Protocol for the improvement of link quality [15]. This algorithm is developed to check the quality of link as a function of SNR. Link with better quality factor are selected for the communication, In this algorithm only good neighbors are kept in routing tables all routes are established over good quality links. This ensures that both broadcast and unicast messages have equal chances of being received correctly regardless of the used transmission rate.

Ionut D. Aron et. al. [16] proposed a localized route repair (LRR) technique, which repairs a route on the fly as soon as it is broken and eliminates the need for network-wide flooding.
LRR technique resulted in an enormous reduction in overhead when simulated on an event driven packet level simulator and results in nearly 30 percent overhead savings. This technique improves other network characteristics as well.

Sergio Crisstomo, et. al. proposes an extension to the AODV protocol, denoted Preemptive Local Route Repair (PLRR) [17], that aims to avoid route failures by preemptively local repairing routes when a link break is about to occur. This protocol is to enhance node's information concerning link stability to its neighbors resorting to HELLO messages. These messages are appended with a mobility extension containing the node's position, motion vector and an associated timestamp. This mobility information will be used to predict the instant $a$ link between two neighbors will break. This proposal does not take into account the sender and destination location information, as other location aided routing protocols.

\section{PROBLEM STATEMENT}

There have been prior works that seek to enhance the basic Local Repair mechanism in order to increase the routing protocol's performance. Some common ideas used in these works include the promiscuous overhearing of control messages from neighboring nodes [12-14] and restricting the local repair search to a small topological radius of the link break $[15,16]$ so that alternative routes to the destination node can be quickly found with minimal routing overheads. However, none of these works have performed a comparative study of the Local Repair and Source Repair mechanisms under different degrees of mobility and network load. In this paper, we explore a flexible, parameterized approach for this decision making process. We consider a range of threshold parameter values for different network scenarios, in particular for different levels of network load. Our simulation results clearly show that a decision making process about route repair strategies that is more flexible and adaptive to the network load level, can lead to a significant performance improvement. The predictive approach will shows that a flexible, parameterized and adaptive approach to choosing the Local Repair Threshold, can improve the Packet Delivery Ratio by up to $37 \%$ (in absolute terms), compared to the approach employed by standard AODV [13]. It also gives a clear description for using such a fixed threshold as a basis for the decision making is suboptimal, since the optimal choice of the threshold depends on the level of network load. This work have demonstrated that an optimal choice of the threshold TLR can result in a significant performance improvement, compared to both the fixed threshold used in AODV, and the Source Repair only approach taken by DYMO [15].

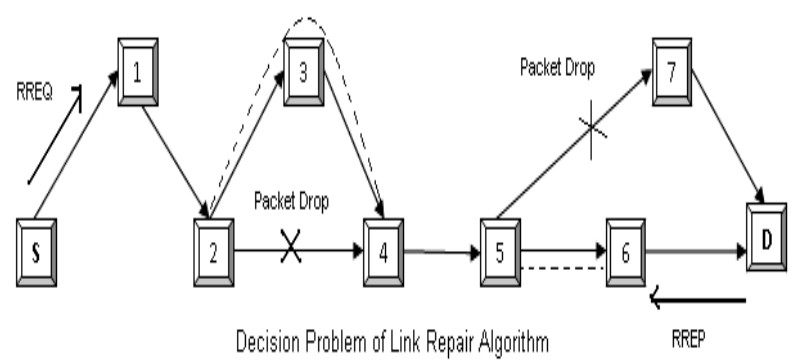

Figure 1:- Link repair decision problem

This paper makes a use of a more flexible, parameterized and adaptive choice of route repair strategies than is implemented by current MANET and Wireless Mesh routing protocols such 
as AODV. In existing AODV protocol the detection of this unpredictable nature of node is not identified as shown in figure 1. Along with that decision of selecting the repairing mechanism is not clarified. While considering the repairing mechanism the link failure gives a triggering of new route discovery mechanism. Thus we required a pre-emptive approach which is been able to detect the link failure before its occurrence [15]. This is done by the link parameters like node energy, link strength, packet delivery ratio etc. Even if the link break occurs than this work will make an active decision of how the algorithm is been selected accordingly. Characteristics of ad hoc networks include resource-poor devices, limited bandwidth, high error rates, and a continually changing topology. Among the available resources, battery power is typically the most constraining. Hence, routing protocol must have Minimal control overhead, Minimal processing overhead, Multi-hop routing capability, Dynamic topology maintenance and Loop prevention. Many proactive and reactive routing are already in existence [17]. The routing tables of the nodes within the neighborhood are organized to optimize response time to local movements and provide quick response time for requests for establishment of new routes. Primary objectives of this algorithm are:

- To broadcast discovery packets only when necessary by failure analysis.

- To distinguish between local connectivity management, neighborhood detection and general topology maintenance.

- To disseminate information about changes in local connectivity to those neighboring mobile nodes that is likely to need the information.

- To Provides Loop Free Route Discovery \& Reputation Free Routes

- To Provide Pre-emptive Route Failure Detection this will identify the link Break Condition early.

- To better take the decision of which route repair mechanism

- To Provide an break free routing which saves cost, Time \& Energy

One of the key open research questions, which these papers have not addressed, is how to determine the optimal Local Repair Threshold (LRT) for different network scenarios and different levels of network load. This is the main focus of our proposed LBD-PTA having multi-hop link failure identification for Local repair in AODV.

\section{PROPOSED MECHANISM}

The proposed mechanism of Link Break Distance (LBD) \& Pre-emptive Threshold Alarm (PTA) is used as a pre-emptive approach to a link break condition \& can also acts as recovery mechanism. It also solves the problem of decision making of which algorithms must be selected at which instance from source repair \& the local repair. According to the proposed algorithm the nodes $\mathrm{S}$ wants to communicate with the destination node $\mathrm{D}$. The source node $\mathrm{S}$ checks the condition whether D is its neighbour node or not. If $\mathrm{D}$ is a neighbour of $\mathrm{S}$ then the route must available. If the route is not available then start new route discovery. If entry is exist in its routing table then the route must be available. Otherwise $\mathrm{S}$ floods a new RREQ packet to its entire neighbour \& flooding continues till the RREQ packet reaches the D node. After receiving the request packet $D$ sends a reply packet of RREP. When $\mathrm{S}$ receives the RREQ it updates its routing table \& communication starts between the S \& D nodes. During this transmission some of the network factors is continuously affecting this data exchange \& will affect or cancel this due to link break. This link break is analysed prior to break occurs from following parameters like motion of node, traffic, battery of node is low, packet delivery ratio is less or time to live (TTL) field is more.

After this analysis we need to calculate a value named as threshold. This must be a base parameter of link to participate or to continue the data transmission. This can also be considered as link strength $\&$ must be measure after a fixed interval of time $(10 \mathrm{sec})$. The value of this threshold needs to be defined which depends on Battery power, Packet delivery Ratio, TTL Value, No of Packet Relayed and Node Mobility within the range.

Threshold $=[$ Initial Battery Power $+($ Packet deliver in Unit Time/ Total number of Packet) + Total Time Taken by Packet +Total number of packet relayed+ Mobile Nodes in a network] * (50/100)

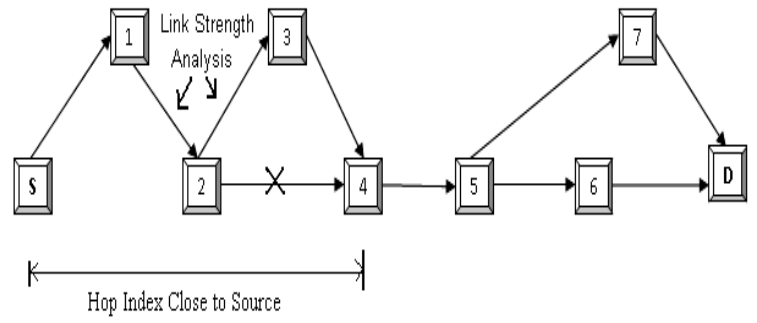

(a) Preemptive Link status analysis \& hop index based source repair

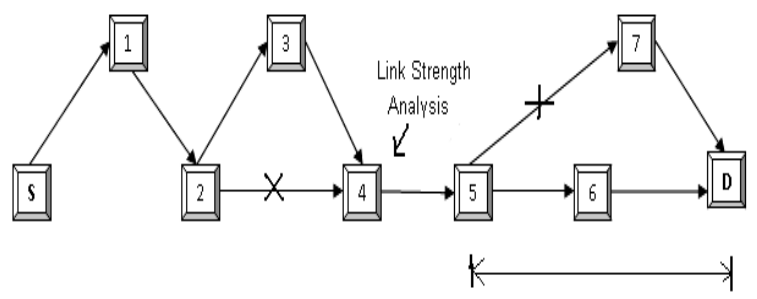

Hop Index Close to Destination

(b) Preemptive Link status analysis \& hop index based Local Repair

Figure 2:- Decision of Applying the source or local repair based on hop index

After measuring the threshold the rank is given to each node in accordance to their strength $\&$ it should be distribute to every node in a range. Thus the routing table updates according to this rank after every 10 seconds. This strength is measure with the above defined threshold value $\&$ checks for two basic condition min (threshold) \& min (min (threshold)). For min (threshold) starts new route discovery again because it is pre-emption to us that the link strength is weaken down in future so new route information must be get ready. But after min (min (threshold) the link break can occurs any time so it is better to shift the transmission to new route rather that losing the data due to failure. This works as an great preemption \& will definitely improves the network performance. Even if the break occurs than we need to calculate the hop index of broken link which gives the link 


\section{Process Flow Structure}

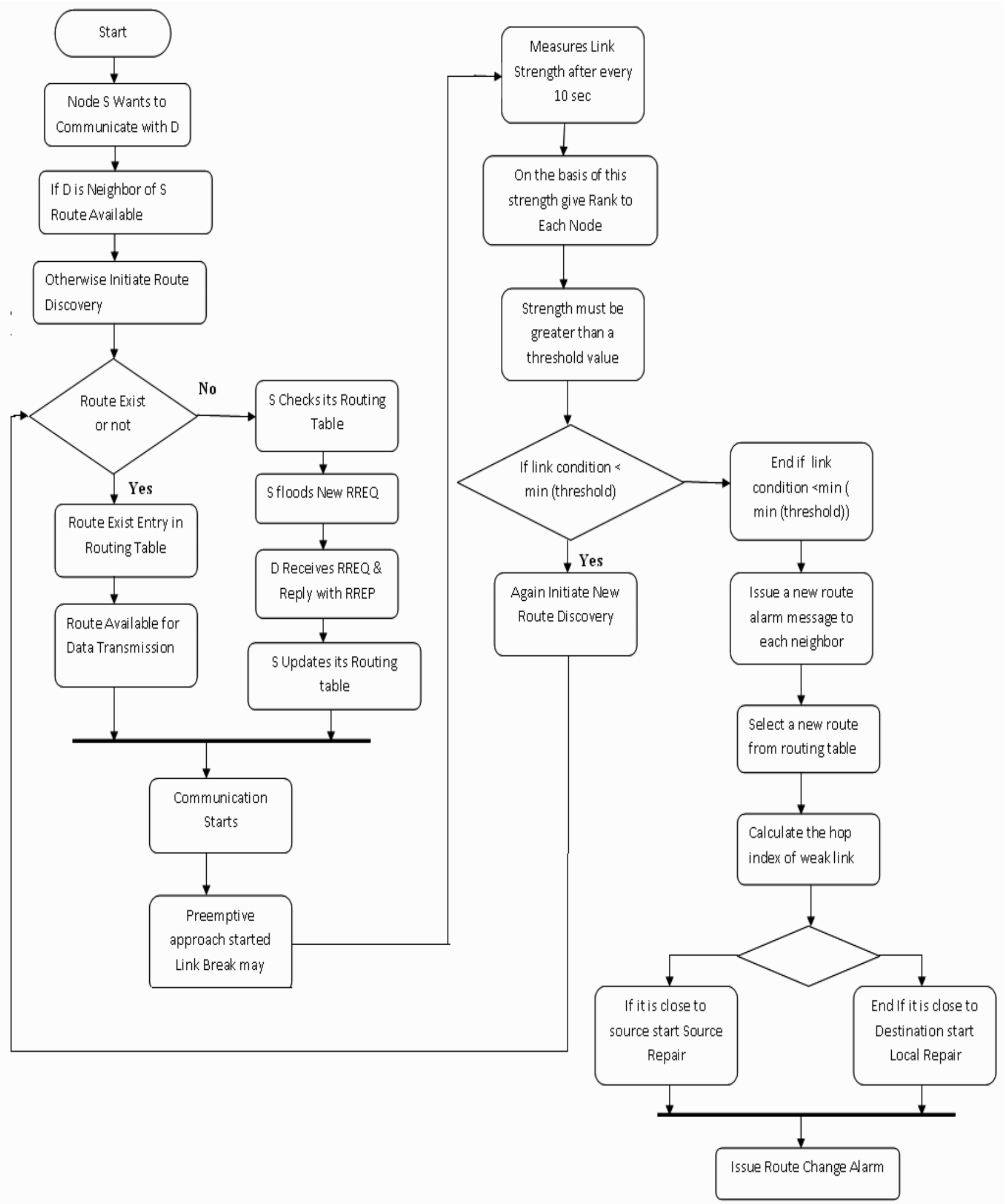

Figure 3:- LBD-PTA Mechanism flow structure of Pre-emptive link repair for AODV 
address which is break down. Now this hop index gives the details that the link break occurs was close to which side source or destination. This gives us the decision making capabilities. If it is close to source then starts source repair algorithm of AODV otherwise Starts Local repair algorithm as given in figure 2 . In this way by the above proposed mechanism of Link Break Distance (LBD) \& Pre-emptive Threshold Alarm (PTA) a fault tolerant routing path is created for data transfer. The detailed description can be understood by process flow structure in Figure 3. (Shown at the end)

Link Break Distance (LBD) \& Pre-emptive Threshold Alarm (PTA) for AODV

1. Source $S$ wants to Communicate with Destination D

2. Route discovery starts in all the four ranges (Quadrant 1, Quadrant 2, Quadrant 3, Quadrant 4)

a. Check If $D$ is a nearest neighbor of $S$ in its routing table

b. If route available link establishes

3. Start new route discovery protocol

4. Source S sends new RREQ (Route Request) to its entire neighbor.

5. This message is flooded till $\mathrm{D}$ receives this.

6. After this D reply with RREP (Route Reply).

7. Routing table entry is updated

8. Each node will send an Empty Hello message to every other node for link status.

9. This link strength is analyzed after every $10 \mathrm{sec}$.

a. This value gives the threshold on the basis of following parameters

b. Threshold (Battery power, Packet delivery Ratio, TTL Value, No of Packet Relayed, Node Mobility within the range)

10. Threshold $=[$ Initial Battery Power $+($ Packet deliver in Unit Time/ Total number of Packet) + Total Time Taken by Packet +Total number of packet relayed+ Mobile Nodes in a network] * $(50 / 100)$

11. Each node shares this information \& gives rank to every other node \& link.

12. If the links condition < min threshold

a. Initiate new route discovery

13. End If threshold $<\min$ (min threshold)

a. Issue Route Change Alarm message to source

b. Each node shares this message \& updates their routing table

14. Even after this pre-emption somewhere link break can occurs due to environmental variable

15. We then investigate what the optimal choice is of this threshold for a range of network scenarios. We define the link break location parameter $\mathbf{l}_{\mathbf{l b}}$ as follows:

$1_{\mathrm{lb}}=$ (hop index of broken link / Total number of hops in Path)
16. This hop index will give the exact location of link on which break occurs.

17. Now calculate the hop count which is close to either source or destination.

18. According to this hop location apply the link repair algorithm

a. If it is close to source apply Source repair

b. If it is close to destination then apply Local Repair

19. Issue an Alarm message to every node

20. Nodes Update their routing table

21. Exit.

\section{CONCLUSION}

The proposed algorithm makes an improvement in terms of both controlling traffic with lowest data loss incurred and resulting gain in path optimality through LBD-PTA. It is a innovative expansion to the modified ADOV protocol so as to achieve better \& pre-emptive link repair. Also the decision making of which repairing mechanism to be used at what condition can also be stimulated through the proposed LBDPTA. The alternative route construction process could be initiated at any time, not just when a route has failed. The dynamically constructed alternative route's information is passed on to the upstream nodes, which then determine by themselves when to direct their packets to the "optimal" alternative route. Thus by applying the threshold based approach and an alarm generation at early stages of link failure will definitely provide the better result in future. Indeed, the proposed scheme detects the best links based on its strength, if any, only between the nodes which are located 2-hop away to each other. Therefore, as a next step, we would like to develop a route repairing \& optimization scheme which is able to detect prior link failures between any pair of nodes on a connection. Finally, such a fault tolerance route repair scheme can lead for effective transmission without worrying about the path optimality \& link condition, which has been addressed as a problem in local recovery operations. At initial level this work proves its efficiency \& will ensures drastic results \& changes in fault tolerant routing in future.

\section{FUTURE WORK}

Some problems and concepts that remain unaddressed can be performed in future. Such as with the help of pre-emptive approach more information can be added for exact timely analysis of link failure. It can also be used for quantitative \& qualitative analysis, rank ordering etc. We also embed source code of our proposed scheme in NS2. In our proposed scheme so as to use the benefits of approach like open source.

\section{ACKNOWLEDGMENTS}

This work will provide an effective link break mechanism for MANET \& cannot be possible to justify the various results without the help of our senior research members. So we provide our sincere thanks to all of them for their great contribution \& guidance. 


\section{REFERENCES}

[1] Saaidal R. Azzuhri, Marius Portmann \& Wee Lum Tan, Evaluation of Parameterised Route Repair in AODV in IEEE Transaction 2011.

[2] Zeki Bilgin \& Bilal Khan, A Dynamic Route Optimization Mechanism for AODV in MANETs in IEEE Transaction 2010.

[3] S. Revathi \& T. R. Rangaswamy , Secured Optimal Adaptable Ad-Hoc Routing Protocol in MANET S. in European Journal of Scientific Research Issn 1450-216, Vol.73 No.1, 2012.

[4] Jyoti Jain, Roopam Gupta in T.K. Bandhopadhyay, On Demand Local Link Repair Algorithm for AODV Protocol in IJCA Volume 35- No.5, December 2011.

[5] Rachid Haboub \& Mohammed Ouzzif, Secure \& reliable routing in MANET in IJCSES Vol.3, No.1, February 2012 .

[6] Nadia Qasim, Fatin Said \& Hamid Aghvami, Mobile Ad Hoc Networking Protocols' Evaluation through Simulation for Quality of Service in IAENG, 36:1, IJCS_36_1_10,February 2009.

[7] P. Priya Naidu1 \& Meenu Chawla, Extended Ad Hoc on Demand Distance Vector Local Repair Trial for MANET in IJWMN, Vol. 4, No. 2, April 2012.

[8] K.Muthumayil, V.Rajamani \& S.Manikandan, Ad-Hoc Cross Layered Energy based on-demand Routing Protocol for MANETs in Conference in R.M.D Engg.College, Gummudipoondi, Dec-2011.

[9] Fikret Sivrikaya, Sahin Albayrak \& Bulent Yener, Spatially Limited Contention for Multi-Hop Wireless Networks in IEEE Globecom Issn 978-1-4244-23248/08 IEEE. 2008.
[10] Zhiming $\mathrm{Xu}$, Yu Wang \& Jingguo Zhu, A Reliable Multicast Routing Protocol for High-speed Mobile Ad Hoc Networks: R-ODMRP in Journal of Software, Vol 9, No 2, 2009

[11] S. Subburam and P. Sheik Abdul Khader, Efficient Two Hop Local Route Repair Mechanism Using Qos-Aware Routing for Mobile Ad Hoc Networks in Indian Journal of Science and Technology ISSN:0974-6846 Vol:5 Issue: 11 November 2012

[12] Hui-Yao An, Ling Zhong, Xi-Cheng Lu, and Wei Peng, A Cluster-Based Multipath Dynamic Source Routing in MANET in IEEE Transaction, 2005.

[13] S. Revathi \& T. R. Rangaswamy , Dynamic Route Shortening and Route Repairing Mechanism for Mobile Ad Hoc Networks in Journal of Computer Science ISSN 1549-3636 8 (8): 1212-1218, 2012.

[14] Jianlin Guo, Chuan Han, Philip Orlik \& Jinyun Zhang, Loop-Free Routing in Low-Power and Lossy Networks in Sensorcomm IARIA, ISBN: 978-1-61208-207-3, 2012.

[15] Srdjan Krco and Marina Dupcinov, Improved Neighbor Detection Algorithm for AODV Routing Protocol IEEE Communications letters, Vol. 7, No. 12, December 2003.

[16] Ionut D. Aron and Sandeep K.S. Gupta, Analytical Comparison of local and End-toEnd Error Recovery in Reactive Routing Protocols for Mobile Ad Hoc Networks, in ACM, MSWIM 2000, pp. 69-76, August 2000.

[17] Sergio Crisstomo , Susana Sargento, edro Brandgo, Rui Prior, Improving AODV with Preemptive Local Route Repair in International Workshop on Wireless Ad-Hoc Networks. 